\title{
EFFECT OF ADVECTED FIELDS ON ACCRETION DISK DYNAMOS
}

\author{
ARUN V. MANGALAM ${ }^{1}$ \\ Department of Physics and Astronomy, Georgia State University, Atlanta, GA 30303-3083 \\ AND \\ K. SUBRAmanian \\ National Centre for Radio Astrophysics, Tata Institute of Fundamental Research, Pune, India \\ Received 1993 March 1; accepted 1993 July 9
}

\begin{abstract}
We present calculations of favored dynamo modes when advection of ambient magnetic fields onto accretion disks is important. These models are relevant for compact binary systems and young stellar objects and can be extended to active galactic nuclei (AGNs). The dynamo equation, including the standard $\alpha$-effect, is modified to take into account advected magnetic fields. Vacuum boundary conditions are assumed outside the disk and the dynamo number switches sign across the equatorial plane. For the local steady state problem, critical dynamo numbers for various modes are obtained analytically. Our motivation is to investigate whether the dominant dynamo generation of quadrupolar magnetic fields and accretion of dipolar magnetic fields is likely to lead to particle acceleration in the form of jets. The results shown here are for a particular choice of boundary conditions and geometry of the advected field. Besides examining other choices, we shall calculate growth rates for different modes, and the influence of the initial seed field configuration on the evolution of the magnetic fields in subsequent work.
\end{abstract}

Subject headings: acceleration of particles — accretion, accretion disks — binaries: close — galaxies: nuclei MHD - stars: pre-main-sequence

\section{INTRODUCTION}

Instances of particle acceleration in the form of bipolar flows are common to many classes of objects including active galactic nuclei, binary X-ray sources, and protostars. Several papers have proposed hydromagnetic mechanisms which utilize strong poloidal magnetic fields emanating from an accretion disk (which is believed to exist around the central object) to drive these flows (Blandford \& Payne 1982; Lovelace, Wang, \& Sulkanen 1987; Pelletier \& Pudritz 1992). The commonality of these mechanisms is that they invoke $v_{p} \| B_{p}$ under steady axisymmetric MHD flow (Chandrasekhar 1956; Mestel 1961).

Our concern in this paper is to examine whether a dynamo operating in the ambience of an advected external field can produce the required geometry for switching on the jets. If indeed these mechanisms are responsible for the jet phenomenon, then the reason for one-sided radio sources seen among AGNs may also be explained within the framework of the acceleration model. Blandford (1989) suggested that a dynamo generating a quadrupolar field in addition to an advected field with dipolar symmetry may produce a significant difference between the poloidal field strength on two sides of the disk leading to a strong and a weak (and hence unseen) jet in a few sources, even though a majority of one-sidedness may be attributed to the relativistic beaming effect. We shall examine this intriguing possibility of a dynamo producing a field of opposite symmetry to that of advected external field.

The dynamo that we shall consider is of the $\alpha-\omega$ variety

\footnotetext{
${ }^{1}$ Visiting Scientist, National Centre for Radio Astrophysics, Tata Institute of Fundamental Research.
}

operating in a Keplerian thin disk. The mean helicity parameter $\alpha$ is given by (Zeldovich, Ruzmaikin, \& Sokoloff 1983, p. 154)

$$
\alpha=l^{2} \boldsymbol{\omega} \cdot \nabla \ln (\rho),
$$

where $l$ is the turbulent length scale, $\omega$ is the angular velocity, and the density scale height $\nabla \ln (\rho)$, will vary in the different regions; also, departures from Keplerian flow will result in dynamos other than the $\alpha-\omega$ type and consequently fields of different symmetry. It will be interesting to study the fields and the global modes resulting from dynamos in the outer region (i.e., galaxy or outer accretion disk) providing the seed field for dynamos operating in the inner region (outer accretion disk or inner accretion disk). Another possible application of this idea could be in accretion disks around neutron stars and X-ray binaries where the star provides a strong ambient dipolar field which may be advected inward and enhanced by long-term dynamo action to produce strong poloidal field lines. In this paper we present calculations of the local steady state problem for a simple case of an ambient dipole field.

\section{MATHEMATICAL FORMULATION}

The standard kinematic dynamo equation has the form (Parker 1955, 1971; Krause \& Rädler 1980; Moffat 1978):

$$
\frac{\partial \boldsymbol{B}}{\partial t}-\nabla \times(\boldsymbol{v} \times \boldsymbol{B}+\alpha \boldsymbol{B})+\nabla \times \eta(\nabla \times \boldsymbol{B})=0,
$$

where $v$ is the laminar velocity, $\eta$ the turbulent resistivity, and $\alpha \boldsymbol{B}$ the " $\alpha$-effect" generating term arising from the helicity of 
the turbulence. Cylindrical polar coordinates $(r, \phi, z)$ are used throughout. The following assumptions and approximations are made: (1) azimuthal symmetry in both velocity and magnetic fields; (2) $\alpha$ is time independent; (3) $\eta$ is taken as uniform; (4) the dynamo is of the $\alpha-\omega$ type, with the effect of differential rotation dominating over the $\alpha$-effect; (5) the thin disk approximation is made, so (Ruzmaikin, Shukurov, \& Sokoloff 1988) $\partial / \partial z \gg \partial / \partial r$; (6) Since we are concerned with the problem of advection the important time scales of the problem are diffusion $\tau_{d}$, and radial drift $\tau_{r}$, and their ratio is given by

$$
\frac{\tau_{r}}{\tau_{d}} \sim \frac{r l v_{t}}{u z^{2}}
$$

where $v_{t}$ is turbulent velocity and $u$ is the radial drift velocity. For the region under consideration in our problem this ratio is large, but we assume that the external field is strong enough that its advection term should be retained in the expansion. We also assume that region is far enough from the central object that relativistic effects of the light cylinder, etc., can be ignored. We introduce the magnetic stream function $\Psi$, which determines the poloidal field strength and is of utility in calculating the mass flux via $v_{p} \propto B_{p}$ :

$$
B_{p}=\left(\frac{1}{r} \nabla \Psi\right) \times \hat{\phi}
$$

Incorporating the above assumptions and using equation (4) we obtain the induction equations for the poloidal magnetic stream function $\Psi$ and the toroidal field $B_{\phi}$ :

$$
\begin{array}{r}
{\left[\frac{\partial}{\partial t}-\eta\left(r \frac{\partial}{\partial r}\left(\frac{1}{r} \frac{\partial}{\partial r}\right)+\frac{\partial^{2}}{\partial z^{2}}\right)+u \frac{\partial}{\partial r}\right] \Psi=\alpha B_{\phi} r} \\
{\left[\frac{\partial}{\partial t}-\eta\left[\frac{\partial}{\partial r}\left(\frac{1}{r} \frac{\partial}{\partial r}\right) r+\frac{\partial^{2}}{\partial z^{2}}\right]+u \frac{\partial}{\partial r}\right] B_{\phi}} \\
=-\frac{\partial \omega}{\partial r} \frac{\partial \Psi}{\partial z}-B_{\phi} \frac{\partial u}{\partial r} .
\end{array}
$$

We shall superpose the external and excited fields in the following manner for mathematical simplification of the problem:

$$
\begin{gathered}
\Psi=\psi+\psi^{e}, \quad B_{\phi}=B \text { for }|z|<h, \text { the disk height; } \\
\Psi=\psi^{v}, \quad B_{\phi}=0 \text { for }|z|=h \\
\psi^{v} \rightarrow \psi^{e} \text { for }|z| \gg h ;
\end{gathered}
$$

where $\psi^{e}$ is the external magnetic field being advected, $\psi$ is the generated field, and $\psi^{v}$ is the intermediate vacuum field at $z=$ $h$ which asymptotically matches to the external field. Since the seed field is curl-free we can use a general form:

$$
\psi^{e}(r, z)=\int a(k) \exp (-k|z|) r J_{1}(k r) d k,
$$

where forms for the spectrum $a(k)$ can be tried out in the global analysis (i.e., variation in $r$ and $z$ ). In this paper we shall do only a local analysis (i.e., variation only in $z$ ) so the boundary conditions (7) $-(9)$ are greatly simplified.

\section{LOCAL ANALYSIS}

We look for critical steady state modes by setting the time derivatives in equations (5)-(6) equal to zero, in which the dissipation is compensated by generation and advection of fields. Neglecting radial diffusion but retaining the radial drift coupled to the external field, applying the approximation (6) and ignoring self advection we get

$$
\begin{gathered}
{\left[\frac{d^{3}}{d z^{3}}+D \alpha(z)\right] B_{r}=-D B_{r}^{e} \alpha(z)-R_{u} \frac{d^{2} B_{z}^{e}}{d z^{2}}} \\
B=\frac{H(z)}{R_{\alpha}}\left(R_{u} B_{z}^{e}+\frac{d B_{r}}{d z}\right)
\end{gathered}
$$

where the dynamo number $D=R_{\alpha} R_{\omega}, R_{\omega}=G h^{2} / \eta, G$ being the shear term and $z$ is scaled by $h . R_{u}$ and $R_{\alpha}=\alpha_{0} h / \eta$ are useful dimensionless numbers. Now we estimate

$$
R_{u}=\frac{h u}{\eta} \approx \frac{h u}{l v_{t}}
$$

and the scaled helicity is taken to be

$$
\alpha(z)=\alpha_{0} H(z), \quad H(z)=\theta(z)-\theta(-z),
$$

where $\theta$ is the step function.

We note that from equation (11) that the particular solution will have no symmetry if $B_{z}^{e}$ and $B_{r}^{e}$ have opposite symmetry and that it will have even (odd) symmetry only when both are odd (even) since the operator acting on $B_{r}$ is odd. The homogeneous solution indeed can have either symmetry, and we therefore note that the radial field can be asymmetric.

The seed field used in this analysis is the classical dipole field expanded to the lowest order terms in $f \equiv h / r$ :

$$
B_{r}^{e}(z)=-3 f z, \quad B_{z}^{e}(z)=-2 f^{2} z^{2}+1
$$

where the $z$ is scaled as before and the magnetic fields are scaled by $B_{z}^{e}(0)$. Since $B_{r}^{e}$ and $B_{z}^{e}$ are not simultaneously odd or even, the general solution has no symmetry and the boundary conditions on both sides of the disk must be included. The general form for the solutions above and below the disk are

$$
\begin{array}{r}
B_{r}^{+}(z)=c_{1} e^{\gamma z}+c_{2} e^{-\gamma z / 2} \sin \left(\frac{\sqrt{3}}{2} \gamma z\right) \\
+c_{3} e^{-\gamma z / 2} \cos \left(\frac{\sqrt{3}}{2} \gamma z\right)+3 f z+4 f^{2} \frac{R_{u}}{D} \\
B_{r}^{-}(z)=c_{4} e^{-\gamma z}+c_{5} e^{\gamma z / 2} \sin \left(\frac{\sqrt{3}}{2} \gamma z\right) \\
+c_{6} e^{\gamma z / 2} \cos \left(\frac{\sqrt{3}}{2} \gamma z\right)+3 f z-4 f^{2} \frac{R_{u}}{D},
\end{array}
$$




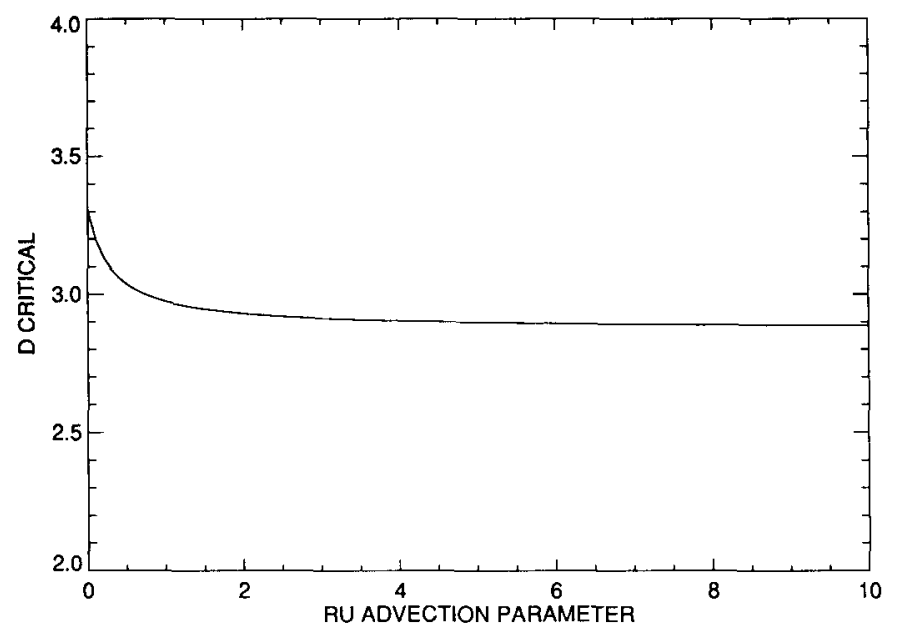

FIG. $1 a$

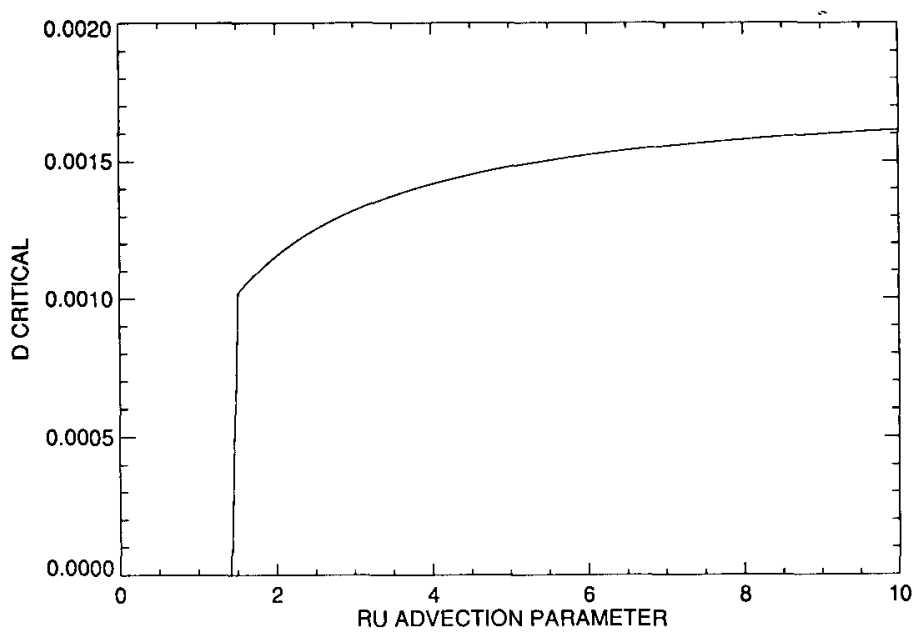

FIG. $1 b$

FIG. 1.- $(a)$ shows the dependence of $R_{u}$, the advection parameter, on the dynamo number for the higher eigenmode and $(b)$, for the lower eigenmode. The lower mode exists only for $R_{u}>1.4$.

where $\gamma=-\sqrt[3]{D}$. The boundary conditions are given by

$$
\left[B_{r}\right](0)=\left[B_{r}^{\prime}\right](0)=\left[B_{r}^{\prime \prime}\right](0)=0
$$

as the third derivative should be defined at 0 , the equator, and similarly we demand that for the azimuthal field:

$$
[B](0)=\left[B^{\prime}\right](0)=0
$$

while they vanish at boundaries: $B( \pm 1)=0$ to match to vacuum fields. The boundary conditions furnish seven algebraic equations for the six unknown coefficients and $\gamma$. Therefore the critical dynamo number can be obtained by numerically solving the equations for a set of inputs of $R_{\alpha}, R_{u}$, and $f$. Note

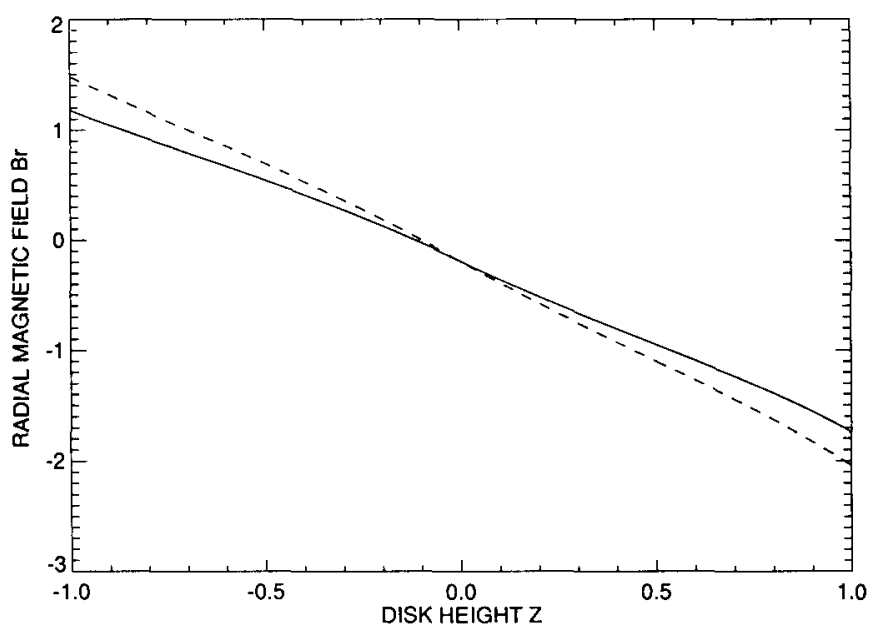

FIG. $2 a$ that the edge conditions on $B_{r}$ cannot be fixed by a purely local analysis. The solution presented here is yielded by a particular choice of boundary conditions adopted, i.e., a smooth match of the fields at the equator. More general boundary conditions, taking into account the radial structure of the fields, will be explored in a detailed paper in preparation (Mangalam \& Subramanian 1994).

\section{RESULTS}

The parameter $R_{\alpha}$ which is of order unity and taken to be 1, merely scales the azimuthal field and hence has no qualitative impact on the nature of solutions. $R_{u}$ given by equation (13) is estimated to be of the order of few typically $\left(v_{t} \sim u\right)$ but can be large close to the central object and negligible near the bound- 


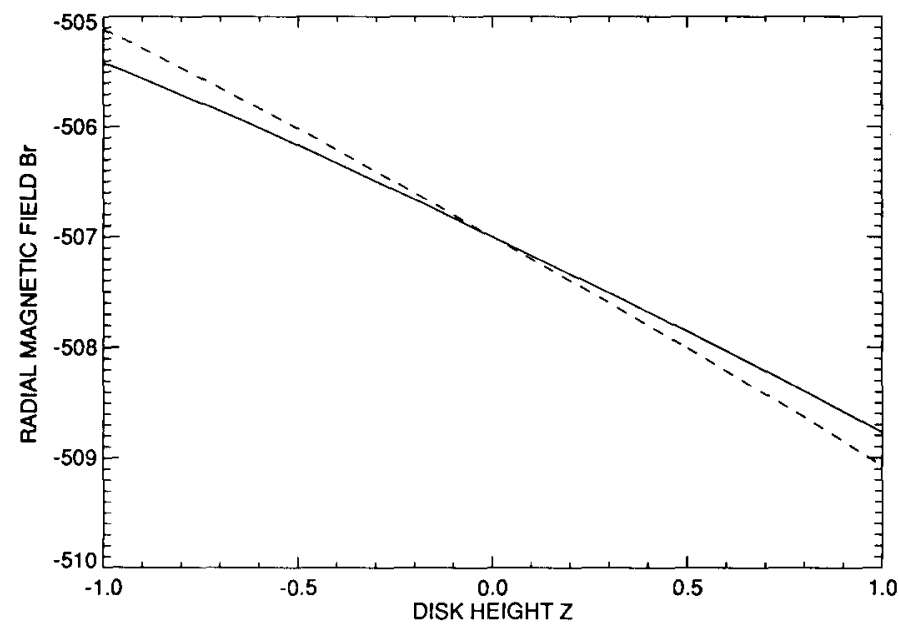

FIG. $3 a$

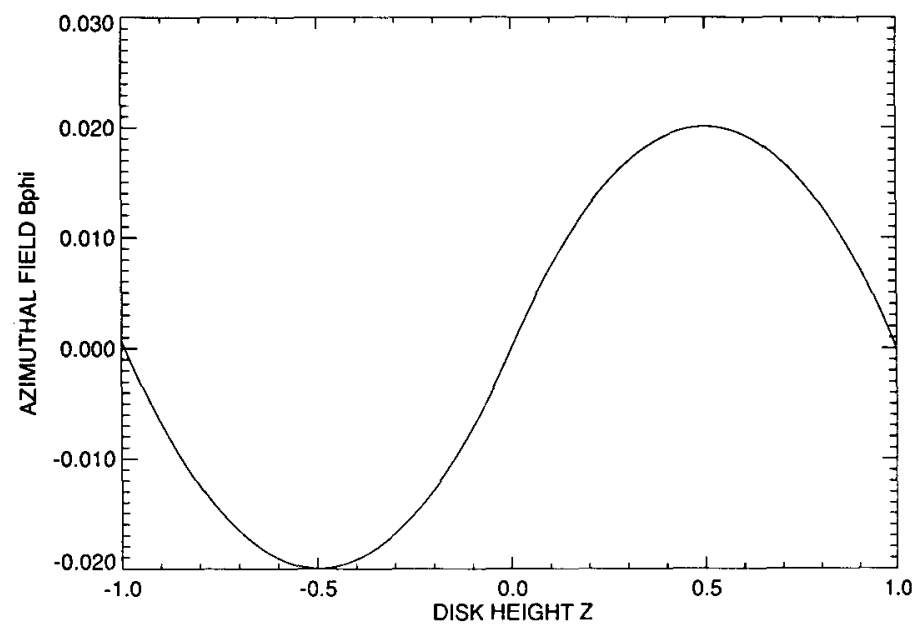

FIG. $3 b$

FIG. 3.- $(a)$ shows the behavior of the radial field (the dashed line is the total field which includes the external field) and ( $b$ ), the behavior of the azimuthal field vs. the height of the disk for the $\gamma=0.1045$ mode. This mode has no symmetry in the radial field and is pointed inward through out the disk.

ary of the disk. The geometry of the external field as determined by $f$ has significant effects toward the center. The region that we consider in our problem has $f<0.1$ since this is a non-relativistic calculation. So we use $R_{u}=[0,10], f=z / r=$ $[0,0.1]$ as the ranges for the inputs; these cover the possible values corresponding to stellar and AGN disks.

The eigenvalue $(\gamma=-\sqrt[3]{D}$.) is solved for by numerically finding the roots resulting from the condition that the determinant of the boundary condition equations is zero. We find that there are basically two modes excited for the parameter space explored: they have $\gamma$ of $\sim 0.1$ and 1.43. The latter mode we find to have very nearly odd symmetries in radial and azimuthal fields, but the former mode has no symmetry in the radial field and is pointed inward throughout the disk. The mode with the lower dynamo number is excited only if $R_{u}$ is greater than a critical value which is $\sim 1.4$ for $f=0.1$; and for $f$ greater than a critical value of 0.08 for $R_{u}$ kept fixed at 2 (see Figs. $1 a-1 b)$. The $(\gamma \approx 1.4)$ mode is universal and can be excited anywhere on the disk and decreases with $f$ and asymptotes to 1.45 for large $R_{u}$.

\section{CONCLUSIONS AND DISCUSSION}

The basic result we find is that the universal mode is easily excited in the inner region (high $f$ and $R_{u}$ ). The other mode has a significantly lower critical value of $D$. The ratio of the amplitudes of the magnetic fields for the two modes are found to be

$$
\frac{B_{r}(\gamma=0.1)}{B_{r}(\gamma=1.43)}=336, \quad \frac{B(\gamma=0.1)}{B(\gamma=1.43)}=0.05,
$$

for inputs of $f=0.1$ and $R_{u}=2$. While both modes lack symmetry as expected, the 1.43 mode is nearly odd, with radial field negative and azimuthal field positive for $z>0$ ( see Figs. 2 and 3 ). This geometry corresponds to the even $z$ symmetry situation given in Lovelace, Wang, \& Sulkanen (1987, their
Fig. 1a) with an oppositely directed current (due to the antisymmetric nature of the toroidal field) and an odd radial magnetic field. The lower eigenmode could very well correspond to the asymmetric case where the radial field is one-sided. The ratios are, however, valid for a steady state and will otherwise vary with time depending on the growth rates of the individual modes. We note that these conclusions from a local analysis cannot be taken too seriously, and one ought to solve the global problem. In particular, the poloidal field geometry which is crucial in the acceleration mechanisms can only be obtained after solving for $B_{z}$.

The expectation in kinematic dynamo theory is that the mode with a lower eigenvalue will also have a higher growth rate, but a situation wherein the back reaction of the fields on the fluid is included is more complicated. We also note that the eigenvalue we have found is lower than the value of $2.45 \mathrm{ob}$ tained for the advection free problem which corresponds to a mode of even symmetry Pudritz (1981a, b). It is likely that the prevalent field may not be completely dynamo generated, but interaction with external fields could help set up the environment for hydromagnetic disk winds.

The global analysis should employ a general form for external curl-free fields in equation (10) so one can experiment with the spectrum. A different form for the helicity (i.e., $\alpha \propto z$; Pudritz 1981b) may be physically more meaningful in accretion disks than the step function used in this preliminary investigation of the problem which forces extra boundary conditions at the equator. It is interesting that the problem can be reversed and solved for forms of $\alpha(z)$ for a given symmetry of the fields (Ruzmaikin \& Sokoloff 1979).

A.M. wishes to thank P. J. Wiita for helpful comments and scrutiny and Gopal-Krishna for enabling his visit to the National Centre for Radio Astrophysics where this work was begun. This research was supported in part by NSF grant AST 9102106 and Smithsonian Institution grant FR 10263600. 


\section{REFERENCES}

Blandford, R. D. 1989, in Theory of Accretion Disks, ed. F. Meyer et al. (Dordrecht: Kluwer), 35

Blandford, R. D., \& Payne, D. G. 1982, MNRAS, 199, 883

Chandrashekar, S. 1956, ApJ, 124, 232

Krause, F., \& Rädler, K. H. 1980, Mean-Field Magnetohydrodynamics and Dynamo Theory (Oxford: Pergamon)

Lovelace, R. V. E., Wang, J. C. L., \& Sulkanen, M. E. 1987, ApJ, 315, 504

Mangalam, A. V., \& Subramanian, K. 1994, in preparation

Mestel, L. 1961, MNRAS, 122, 473

Moffat, H. K. 1978, Magnetic Field Generation in Electrically Conducting Fluids (Cambridge Univ. Press)
Parker, E. N. 1955, ApJ, 122, 293

- 1971, ApJ, 163, 255

Pelletier, G., \& Pudritz, R. E., 1992, ApJ, 394, 117

Pudritz, R. E. 1981a, MNRAS, 195, 881

1981b, MNRAS, 195, 897

Ruzmaikin, A. A., Shukurov, A. M., \& Sokoloff, D. D. 1988, Magnetic Fields of Galaxies (Dordrecht: Kluwer)

Ruzmaikin, A. A., \& Sokoloff, D. D. 1979, A\&A, 78, 1

Zeldovich, Y. B., Ruzmaikin, A. A., \& Sokoloff, D. D. 1983, Magnetic Fields in Astrophysics (New York: Gordon \& Breach) 